\title{
Large Deviations for Stationary Gaussian Processes*
}

M. D. Donsker and S. R. S. Varadhan

Courant Institute of Mathematical Sciences, New York University, New York, NY 10012, USA

\begin{abstract}
In their previous work on large deviations the authors always assumed the base process to be Markovian whereas here they consider the base process to be stationary Gaussian. Similar large deviation results are obtained under natural hypotheses on the spectral density function of the base process. A rather explicit formula for the entropy involved is also obtained.
\end{abstract}

\section{Introduction}

Let $\left\{X_{k}\right\},-\infty<k<\infty$, be a stationary Gaussian process with $E\left\{X_{k}\right\}=0$ and $E\left\{X_{0} X_{j}\right\}=\varrho_{j}=\frac{1}{2 \pi} \int_{0}^{2 \pi} e^{i j \theta} f(\theta) d \theta$. We assume that the spectral density function $f(\theta)$ is continuous on $[0,2 \pi], f(0)=f(2 \pi)$, and

$$
\int_{0}^{2 \pi} \log f(\theta) d \theta>-\infty .
$$

Let $\Omega=\prod_{j=-\infty}^{\infty} \mathbb{R}_{j}$ where, for each $j, \mathbb{R}_{j}$ is the real line, i.e., $\Omega$ is the space of doubly infinite sequences of real numbers. We specify a point $\omega \in \Omega$ by $\omega=\left\{x_{k}\right\}$, $-\infty<k<\infty$, and let $\omega(j)=x_{j}$ for $-\infty<j<\infty$. The process $\left\{X_{k}\right\}$ induces a probability measure $P$ on $\Omega$. We will denote integration over $\Omega$ with respect to $P$ measure by $E^{P}\{\}$.

For each positive integer $n$ and each $\omega \in \Omega$, let $\omega^{(n)}$ be the point in $\Omega$ obtained by the periodic extension in both directions of the elements $x_{1}, x_{2}, \ldots, x_{n}$ of $\omega$, i.e., if $\omega=\left\{x_{k}\right\},-\infty<k<\infty$, then $\omega^{(n)}$ is the point

$$
\ldots, x_{1}, \ldots, x_{n-1}, x_{n}, x_{1}, x_{2}, \ldots, x_{n}, x_{1}, x_{2}, \ldots, x_{n}, \ldots
$$

\footnotetext{
* The research in this paper was supported by the National Science Foundation, Grant No. MCS-80-02568
} 
Let $T$ be the shift operator acting on the points of $\Omega$. For each $\omega \in \Omega$ and each positive integer $n$, we define a probability measure on $\Omega$, call it $\pi_{n}(\omega)$, as follows:

$$
\pi_{n}(\omega)=\frac{1}{n}\left(\delta_{\omega^{(n)}}+\delta_{T \omega^{(n)}}+\ldots+\delta_{\left.T^{n-1} \omega^{(n)}\right)} .\right.
$$

In other words, given $\omega \in \Omega$ and $n \geqq 1, \pi_{n}(\omega)$ is the probability measure on $\Omega$ which assigns mass $1 / n$ to $\omega^{(n)}$ and to each of its $(n-1)$ translates. We note that $\pi_{n}(\omega)$ is a translation invariant measure on $\Omega$, i.e., a stationary measure on $\Omega$. Let $\underline{M}_{S}$ be the space of all stationary measures on $\Omega$ and impose on $\underline{M}_{S}$ the topology of weak convergence. We can use the mapping $\pi_{n}: \Omega \rightarrow \underline{M}_{S}$ and the measure $P$ on $\Omega$ to construct a probability measure on $\underline{M}_{S}$ by defining, for each $n, Q_{n}=\pi_{n} P^{-1}$, i.e., if $A$ is a set of stationary measures in $\underline{M}_{S}$, then

$$
Q_{n}(A)=P\left\{\omega \in \Omega: \pi_{n}(\omega) \in A\right\} .
$$

The assumptions we made on the Gaussian process $\left\{X_{k}\right\}$ imply that it is an ergodic process, and it then follows from the ergodic theorem that, for almost all $\omega$ ( $P$-measure), the measure $\pi_{n}(\omega)$ converges weakly to the measure $P$ as $n \rightarrow \infty$, i.e.,

$$
P\left\{\omega \in \Omega: \pi_{n}(\omega) \Rightarrow P\right\}=1 .
$$

Thus, with reference to the $Q_{n}$ measure on $\underline{M}_{S}$ given by (1.3), we expect that if the set $A$ contains the measure $P$, then $Q_{n}(A) \rightarrow 1$ as $n \rightarrow \infty$, whereas if $P$ is not in the closure of $A$, we expect $Q_{n}(A) \rightarrow 0$ as $n \rightarrow \infty$. In this paper we show that in this latter case $Q_{n}(A)$ approaches zero exponentially fast as $n \rightarrow \infty$ and we determine the constant in the exponential rate. To be more specific, let $R$ denote a general element of $\underline{M}_{S}$, i.e., a stationary measure on $\Omega$, and recall that $f$ is the spectral density of our basic stationary Gaussian process $\left\{X_{k}\right\}$. We define a functional $H_{f}(R)$ which is, in fact, the entropy of the stationary process $R$ with respect to the stationary Gaussian process $\left\{X_{k}\right\}$, and $H_{f}(R): \underline{M}_{S} \rightarrow[0, \infty]$ is such that:

If $C \subset M_{S}$ is closed,

and if $G \subset \underline{M}_{S}$ is open,

$$
\varlimsup_{n \rightarrow \infty} \frac{1}{n} \log Q_{n}(C) \leqq-\inf _{R \in C} H_{f}(R),
$$

$$
\varliminf_{n \rightarrow \infty} \frac{1}{n} \log Q_{n}(G) \geqq-\inf _{R \in G} H_{f}(R) .
$$

Consistent with remarks made above it will indeed be true that $H_{f}(P)=0$. We give an explicit formula for $H_{f}(R)$ in (1.9) below.

Let $\Phi: \underline{M}_{S} \rightarrow \mathbb{R}$ be bounded and continuous, and let $E^{Q_{n}}\{\}$ denote integration over $\underline{M}_{S}$ with respect to $Q_{n}$ measure. From (1.5) and (1.6) it follows easily that

$$
\lim _{n \rightarrow \infty} \frac{1}{n} \log E^{Q_{n}}\left\{e^{n \Phi(R)}\right\}=\sup _{R \in \underline{M} S}\left[\Phi(R)-H_{f}(R)\right] .
$$

Since $Q_{n}=\pi_{n} P^{-1}$, equivalent to (1.7) is

$$
\lim _{n \rightarrow \infty} \frac{1}{n} \log E^{P}\left\{e^{n \Phi\left(\pi_{n}(\omega)\right)}\right\}=\sup _{R \in M_{S}}\left[\Phi(R)-H_{f}(R)\right] .
$$


The main results of this paper then are (1.5), (1.6), their implication (1.8), and the formula (1.9) for $H_{f}(R)$ which we discuss now.

Let $R \in \underline{M}_{S}$ and let, for $A \subset \mathbb{R}, R(A \mid \omega)=R\left(X_{0} \in A \mid X_{-1}, X_{-2}, \ldots\right)$ be the regular conditional probability distribution of $X_{0}$ given the entire past. Denote by $r(y \mid \omega)$ the corresponding density. If $G(\theta)$ is the spectral measure of the stationary process $R$, then the formula for $H_{f}(R)$ is

$$
\begin{aligned}
H_{f}(R)= & E^{R}\left\{\int_{-\infty}^{\infty} r(y \mid \omega) \log r(y \mid \omega) d y\right\} \\
& +\frac{1}{2} \log 2 \pi+\frac{1}{4 \pi} \int_{0}^{2 \pi} \frac{d G(\theta)}{f(\theta)}+\frac{1}{4 \pi} \int_{0}^{2 \pi} \log f(\theta) d \theta,
\end{aligned}
$$

where it is understood that $H_{f}(R)$ is defined to be $+\infty$ if for any reason we cannot define any of the ingredients in (1.9).

The authors have developed a theory of large deviations for Markov processes (see [1, 2], for theoretical results and e.g. [3-5] for some applications thereof). In theory, our methods should apply to rather general stationary processes, but it is difficult to see in any great generality what the natural hypotheses to impose on a stationary process are in order to obtain the analogues of (1.5),(1.6), and (1.8), much less to obtain the analogue of (1.9) in anything like explicit form. For recent work in this direction see Orey [6]. In this paper we consider the special case of a Gaussian stationary process because as seen above the hypotheses needed are natural and we obtain an explicit formula for the corresponding entropy.

In Sect. 2 of this paper we prove (1.5) and (1.6) but in doing so we must use properties of $H_{f}(R)$ and other entropies which occur in the proofs. So as not to interrupt the argument we prove these properties in Sect. 3.

\section{Proofs of the Main Theorems}

As in the introduction, let $\left\{X_{k}\right\}$ be a stationary Gaussian process and $E\left\{X_{0} X_{j}\right\}=\varrho_{j}$ $=\frac{1}{2 \pi} \int_{0}^{2 \pi} e^{i j \theta} f(\theta) d \theta$, where we assume that the spectral density function $f(\theta)$ is continuous on $[0,2 \pi], \mathrm{f}(0)=\mathrm{f}(2 \pi)$, and $\int_{0}^{2 \pi} \log f(\theta) d \theta>-\infty$.

Let $\left\{\xi_{k}\right\},-\infty<k<\infty$, be a sequence of independent Gaussian random variables each with mean 0 and variance 1 . If we let $\left\{a_{n}\right\}$ be the Fourier coefficients of $\sqrt{f(\theta)}$, i.e., $\sqrt{f(\theta)}=\sum_{n=-\infty}^{\infty} a_{n} e^{i n \theta}$, then we can write

$$
X_{k}=\sum_{n=-\infty}^{\infty} a_{n-k} \xi_{k}=\sum_{n=-\infty}^{\infty} a_{n} \xi_{n+k} .
$$

Let $N$ be a positive integer and define

$$
h_{N}(\theta)=\sum_{|j|<N} a_{j}\left(1-\frac{|j|}{N}\right) e^{i j \theta},
$$


where the $a_{j}$ 's are defined in the preceding paragraph. Since $f(\theta)$ is continuous and $f(0)=f(2 \pi)$, we have by Fejer's theorem,

$$
\lim _{N \rightarrow \infty} \sup _{0 \leqq \theta \leqq 2 \pi}\left|h_{N}(\theta)-\sqrt{f(\theta)}\right|=0 .
$$

For each $N$, define the process

$$
X_{k}^{N}=\sum_{|j|<N} a_{j}\left(1-\frac{|j|}{N}\right) \xi_{j+k} .
$$

If we let $f_{N}(\theta)=h_{N}^{2}(\theta)$, then $f_{N}(\theta)$ is the spectral density of the process $\left\{X_{k}^{N}\right\}$.

In the introduction we noted that the process $\left\{X_{k}\right\}$ induces a probability measure $P$ on $\Omega$. Similarly the process $\left\{X_{k}^{N}\right\}$ just defined induces a probability measure $P^{N}$ on $\Omega$. Indeed, the family $\left\{\xi_{k}\right\}$ of independent, Gaussian, mean 0 , variance 1 , random variables itself induces a probability measure on $\Omega$ which we will denote by $\mu$. Each of these three stationary measures on $\Omega$ together with the mapping $\pi_{n}: \Omega \rightarrow \underline{M}_{S}$ of the introduction generates a probability measure on $\underline{M}_{S}$. We have already introduced $Q_{n}=P \pi_{n}^{-1}$. Analogously, we define $v_{n}=\mu \pi_{n}^{-1}$ and for each $N, Q_{n}^{N}=P^{N} \Pi_{n}^{-1}$.

For convenience let $b_{k}=a_{k}\left(1-\frac{|k|}{N}\right)$ and define the mapping $F_{N}: \Omega \rightarrow \Omega$ by

$$
\left(F_{N}(\omega)\right)(j)=\sum_{|k|<N} b_{k} x_{j+k},
$$

so that $F_{N}$ is a moving average map of $\Omega$ into itself. The mapping $F_{N}: \Omega \rightarrow \Omega$ induces a corresponding map $\tilde{F}_{N}: \underline{M}_{S} \rightarrow \underline{M}_{S}$.

We also have the mapping $F: \Omega \rightarrow \Omega$ given by

$$
(F(\omega))(j)=\sum_{k=-\infty}^{\infty} a_{k} x_{j+k},
$$

which exists for almost all $\omega(\mu$ measure on $\Omega)$, since $\sum_{n=-\infty}^{\infty} a_{n}^{2}<\infty$. We note that for sets $A \subset \underline{M}_{S}$ :

$$
\begin{gathered}
Q_{n}(A)=P\left\{\omega \in \Omega: \pi_{n}(\omega) \in A\right\}=\mu\left\{\omega \in \Omega: \pi_{n} \cdot F(\omega) \in A\right\}, \\
Q_{n}^{N}(A)=P^{N}\left\{\omega \in \Omega: \pi_{n}(\omega) \in A\right\}=\mu\left\{\omega \in \Omega: \pi_{n} \cdot F_{N}(\omega) \in A\right\}, \\
v_{n}(A)=\mu\left\{\omega \in \Omega: \pi_{n}(\omega) \in A\right\} .
\end{gathered}
$$

It will be important to introduce also the probability measure $\widetilde{Q}_{n}^{N}$ on $\underline{M}_{S}$ given by $\widetilde{Q}_{n}^{N}=v_{n} \widetilde{F}_{n}^{-1}$ so that

$$
\tilde{Q}_{n}^{N}(A)=v_{n}\left\{R \in \underline{M}_{S}: \tilde{F}_{N}(R) \in A\right\}=\mu\left\{\omega \in \Omega: \tilde{F}_{N} \cdot \pi_{n}(\omega) \in A\right\} .
$$

To deduce the asymptotic behavior of $Q_{n}$ as $n \rightarrow \infty$, i.e., to prove (1.5) and (1.6), we first determine the asymptotic behavior of $Q_{n}^{N}$ as $n \rightarrow \infty$ and then let $N \rightarrow \infty$. To obtain the asymptotics of $Q_{n}^{N}$ we compare it with $\widetilde{Q}_{n}^{N}$ the asymptotic behavior of which as $n \rightarrow \infty$ follows from our earlier work and which we state below as Theorem 2.2. Looking at (2.8) and (2.7) we see that to compare $\widetilde{Q}_{n}^{N}$ and $Q_{n}^{N}$ means 
comparing $\pi_{n} \cdot F_{N}(\omega)$ with $\tilde{F}_{N} \cdot \pi_{n}(\omega)$. Now,

$$
\tilde{F}_{N} \cdot \pi_{n}(\omega)=\frac{1}{n}\left[\delta_{F_{N}\left(\omega^{(n)}\right)}+\delta_{F_{N}\left(T \omega^{(n)}\right)}+\ldots+\delta_{F_{N}\left(T^{n-1} \omega^{(n)}\right)}\right]
$$

and

$$
\pi_{n} \cdot F_{N}(\omega)=\frac{1}{n}\left[\delta_{\left(F_{N}(\omega)\right)^{(n)}}+\delta_{T\left(F_{N}(\omega)\right)^{(n)}}+\ldots+\delta_{T^{n-1}\left(F_{N}(\omega)\right)^{(n)}}\right]
$$

Thus, to compare the two measures $\tilde{F}_{N} \cdot \pi_{n}$ and $\pi_{n} \cdot F_{N}$, we need to determine when $T^{j-1}\left(F_{N}(\omega)\right)^{(n)}=F_{N}\left(T^{j-1} \omega^{(n)}\right)$ with $1 \leqq j \leqq n$. It is clear that the difference of the two measures $\pi_{n} \cdot F_{N}$ and $\widetilde{F}_{N} \cdot \pi_{n}$ can be estimated in the variation norm over the $\sigma$-field generated by the first $L$ components. We obtain

$$
\left\|\pi_{n} \cdot F_{N}-\tilde{F}_{N} \cdot \pi_{n}\right\|_{F_{L}^{\prime}} \leqq \frac{2 N+L}{n} .
$$

In the space $\underline{M}_{S}$ we have the topology of weak convergence. This topology can be induced by the following metric. Let $R_{1}$ and $R_{2}$ be in $\underline{M}_{S}$ and let $L$ be a positive integer. Denote by $R_{1}^{L}$ and $R_{2}^{L}$ the respective $L$-dimensional measures one obtains by considering the joint distribution of $L$ successive random variables in the processes $R_{1}$ and $R_{2}$. We then define the distance between $R_{1}$ and $R_{2}$ in $\underline{M}_{S}$ by

$$
d\left(R_{1}, R_{2}\right)=\sum_{L=1}^{\infty} \frac{1}{2^{L}} d_{L}\left(R_{1}^{L}, R_{2}^{L}\right)
$$

where $d_{L}$ is the Prohorov distance for $L$-dimensional measures. This metric in $\underline{M}_{S}$ yields the topology of weak convergence in $\underline{M}_{S}$. We now can prove the following lemma.

Lemma 2.1. For any $\delta>0$ and large enough $n$,

$$
\mu\left\{\omega \in \Omega: d\left(\pi_{n} \cdot F_{N}, \tilde{F}_{N} \cdot \pi_{n}\right) \geqq \varepsilon\right\}=0 .
$$

Proof. From (2.12), (2.11), and the fact that the variation norm dominates the Prohorov norm in $L$-dimensions, we have for $n>N$,

$$
d\left(\pi_{n} \cdot F_{N}, \tilde{F}_{n} \cdot \pi_{n}\right) \leqq \sum_{L=1}^{\infty} \frac{1}{2^{L}}\left\|\pi_{n} \cdot F_{N}-\tilde{F}_{N} \cdot \pi_{n}\right\|_{\underline{F}_{L}^{\prime}} \leqq \frac{1}{n} \sum_{L=1}^{\infty} \frac{2 N+L}{2^{L}} .
$$

Since the infinite series on the right converges, we can make the distance on the left as small as we please by taking $n$ large enough which implies (2.13).

As noted in (2.8),

$$
\tilde{Q}_{n}^{N}(A)=v_{n}\left\{R \in \underline{M}_{S}: \widetilde{F}_{N}(R) \in A\right\}=\mu\left\{\omega \in \Omega: \tilde{F}_{N} \cdot \pi_{n}(\omega) \in A\right\} .
$$

Since $\mu$ measure on $\Omega$ comes from the sequence $\left\{\xi_{k}\right\}$ of independent Gaussian variables with mean 0 and variance 1 , the results of [2-IV] give us the asymptotics of $v_{n}$ measure on $\underline{M}_{S}$ and hence of $\widetilde{Q}_{n}^{N}$ measure on $\underline{M}_{S}$. 
In the introduction we defined for $R \in \underline{M}_{S}$ the density $r(y \mid \omega)$ of the regular condition probability distribution of $X_{0}$ given the entire past. For $R \in \underline{M}_{S}$ define

$$
I(R)=E^{R}\left\{\int_{-\infty}^{\infty} r(y \mid \omega) \log \left(\frac{r(y \mid \omega)}{\frac{1}{\sqrt{2 \pi}} e^{-y^{2} / 2}}\right) d y\right\},
$$

and define for $R \in \underline{M}_{S}$

$$
H_{f_{N}}(R)=\inf _{R^{\prime} \in \underline{M} S: R^{\prime} \widetilde{F}_{\bar{N}}^{1}=R} I\left(R^{\prime}\right) .
$$

Then in Sect. 3, formula (3.21), we show

$$
\begin{aligned}
H_{f_{N}}(R)= & E^{R}\left\{\int_{-\infty}^{\infty} r(y \mid \omega) \log r(y \mid \omega) d y\right\}+\frac{1}{2} \log 2 \pi \\
& +\frac{1}{4 \pi} \int_{0}^{2 \pi} \frac{d G(\theta)}{f_{N}(\theta)}+\frac{1}{4 \pi} \int_{0}^{2 \pi} \log f_{N}(\theta) d \theta,
\end{aligned}
$$

where $G(\theta)$ is the spectral measure of $R$ and $f_{N}(\theta)$ (see just after (2.4)) is the spectral density of the $\left\{X_{R}^{N}\right\}$ process. have

Thus, from [2-IV] and the contraction principle discussed in that paper, we

Theorem 2.2. If $C \subset \underline{M}_{S}$ is closed,

$$
\varlimsup_{n \rightarrow \infty} \frac{1}{n} \log \widetilde{Q}_{n}^{N}(C) \leqq-\inf _{R \in C} H_{f_{N}}(R),
$$

and if $G \subset \underline{M}_{S}$ is open,

$$
\varliminf_{n \rightarrow \infty} \frac{1}{n} \log \tilde{Q}_{n}^{N}(G) \geqq-\inf _{R \in G} H_{f_{N}}(R) .
$$

Using this and Lemma 2.1 we can prove the following theorem.

Theorem 2.3. If $C \subset \underline{M}_{S}$ is closed,

$$
\varlimsup_{n \rightarrow \infty} \frac{1}{n} \log Q_{n}^{N}(C) \leqq-\inf _{R \in C} H_{f_{N}}(R),
$$

and if $G \subset \underline{M}_{S}$ is open,

$$
\varliminf_{n \rightarrow \infty} \frac{1}{n} \log Q_{n}^{N}(G) \geqq-\inf _{R \in G} H_{f_{N}}(R) .
$$

Proof. Let $\delta>0$ and $C^{\delta}=\bigcup_{R \in C} S(R, \delta)$ where $S(R, \delta)$ is the ball in $\underline{M}_{S}$ of radius $\delta$ centered at $R$. From (2.7) and (2.8) we see that

$$
\begin{aligned}
Q_{n}^{N}(C) & =\mu\left\{\omega \in \Omega: \pi_{n} \cdot F_{N}(\omega) \in C\right\} \\
& \leqq \mu\left\{\omega \in \Omega: \widetilde{F}_{N} \cdot \pi_{n}(\omega) \in C^{\delta}\right\}+\mu\left\{\omega \in \Omega: d\left(\pi_{n} \cdot F_{N}, \widetilde{F}_{n} \cdot \pi_{n}\right) \geqq \delta\right\} \\
& =\widetilde{Q}_{n}^{N}\left(C^{\delta}\right)+\mu\left\{\omega \in \Omega: d\left(\pi_{n} \cdot F_{N}, \widetilde{F}_{N} \cdot \pi_{n}\right) \geqq \delta\right\} .
\end{aligned}
$$


Let $\bar{C}^{\delta}$ be the closure of $C^{\delta}$. Then, from Lemma 2.1 and Theorem 2.2,

$$
\varlimsup_{n \rightarrow \infty} \frac{1}{n} \log Q_{n}^{N}(C) \leqq \varlimsup_{n \rightarrow \infty} \frac{1}{n} \log \widetilde{Q}_{n}^{N}\left(\bar{C}^{\delta}\right) \leqq-\inf _{R \in \bar{C}^{\delta}} H_{f_{N}}(R) .
$$

Since $C$ is closed, $H_{f_{N}}(R)$ is lower semicontinuous, and $\left\{R: H_{f_{N}}(R) \leqq K\right\}$ is tight, (cf. Lemma 3.10) we obtain (2.18) from (2.20) on letting $\delta \rightarrow 0$.

For the lower bound, let $G$ be open in $\underline{M}_{S}$ and let $R \in G$. Pick $\delta>0$ and an open set $V$ such that $R \in V \subset V^{\delta} \subset G$. Then, again noting (2.7) and (2.8),

$$
\begin{aligned}
Q_{n}^{N}(G) & \geqq Q_{n}^{N}\left(V^{\delta}\right)=\mu\left\{\omega \in \Omega: \pi_{n} \cdot F_{N}(\omega) \in V^{\delta}\right\} \\
& \geqq \mu\left\{\omega \in \Omega: \widetilde{F}_{N} \cdot \pi_{n}(\omega) \in V\right\}-\mu\left\{\omega \in \Omega: d\left(\pi_{n} \cdot F_{N}, \widetilde{F}_{N} \cdot \pi_{n}\right) \geqq \delta\right\} \\
& =\widetilde{Q}_{n}^{N}(V)-\mu\left\{\omega \in \Omega: d\left(\pi_{n} \cdot F_{N}, \widetilde{F}_{N} \cdot \pi_{n}\right) \geqq \delta\right\} .
\end{aligned}
$$

Using again Lemma 2.1 and Theorem 2.2,

$$
\varliminf_{n \rightarrow \infty} \frac{1}{n} \log Q_{n}^{N}(G) \geqq \varliminf_{n \rightarrow \infty} \frac{1}{n} \log \widetilde{Q}_{n}^{N}(V) \geqq-\inf _{R^{\prime} \in V} H_{f_{N}}(R) \geqq-H_{f_{N}}(R) .
$$

Since inequality (2.21) holds for any $R \in G$ we obtain (2.19).

Before proving the next theorem we need some preliminary lemmas.

Lemma 2.4. Let $\left\{X_{k}\right\}$ and $\left\{X_{k}^{N}\right\}$ be defined respectively by (2.1) and (2.4). There exists a function $\varepsilon(N)=o(1)$ as $N \rightarrow \infty$ such that if $0<\lambda<\frac{1}{2 \varepsilon(N)}$, then for all $n$,

$$
\frac{1}{n} \log E^{\mu}\left\{\exp \left\{\lambda \sum_{k=1}^{n}\left(X_{k}-X_{k}^{N}\right)^{2}\right\}\right\} \leqq-\frac{1}{2} \log (1-2 \lambda \varepsilon(N)) .
$$

Proof. First of all, let $\left\{Y_{k}\right\}$ be any stationary Gaussian process with mean 0 , covariance $\gamma_{j}=\frac{1}{2 \pi} \int_{0}^{2 \pi} e^{i j \theta} g(\theta) d \theta$, and strictly positive definite covariance matrix

$$
A_{n}=\left(\begin{array}{cccc}
\gamma_{0} & \gamma_{1} & \ldots & \gamma_{n-1} \\
\gamma_{1} & \gamma_{0} & \ldots & \gamma_{n-2} \\
\ldots & \ldots & \ldots \\
\gamma_{n-1} & \cdots & \gamma_{0}
\end{array}\right) .
$$

Let $c=\sup _{0 \leqq \theta \leqq 2 \pi}|g(\theta)|$. We show now that if $\lambda<\frac{1}{2 c}$, then

$$
E\left\{\exp \left\{\lambda \sum_{k=1}^{n} Y_{k}^{2}\right\}\right\} \leqq(1-2 \lambda c)^{-n / 2} .
$$

To see this, first observe that if $c_{n}$ is the maximum eigenvalue of $A_{n}$,

$$
\begin{aligned}
E\left\{\exp \left\{\lambda \sum_{k=1}^{n} Y_{k}^{2}\right\}\right\} & =\frac{1}{\sqrt{\left|A_{n}\right|}} \frac{1}{(2 \pi)^{n / 2}} \int_{-\infty}^{\infty} \ldots \exp \left\{\lambda \sum_{k=1}^{n} y_{k}^{2}-\frac{1}{2} Y^{\prime} A_{n}^{-1} Y\right\} d y_{1} \ldots d y_{n} \\
& =\frac{1}{\sqrt{\left|A_{n}\right|}} \frac{1}{\sqrt{\left|A_{n}^{-1}-2 \lambda I\right|}}=\frac{1}{\sqrt{\left|I-2 \lambda A_{n}\right|}} \leqq\left(1-2 \lambda c_{n}\right)^{-n / 2}
\end{aligned}
$$


providing $\lambda<1 / 2 c_{n}$. To estimate $c_{n}$, consider

$$
\sum_{j, k=1}^{n} \gamma_{k-j} \eta_{k} \bar{\eta}_{j}=\frac{1}{2 \pi} \int_{0}^{2 \pi}\left|\sum_{k=1}^{n} \eta_{k} e^{i k \theta}\right|^{2} g(\theta) d \theta \leqq \sup _{0 \leqq \theta \leqq 2 \pi}|g(\theta)| \sum_{j=1}^{n}\left|\eta_{j}\right|^{2}
$$

which implies $c_{n} \leqq \sup _{0 \leqq \theta \leq 2 \pi}|g(\theta)|=c$. Thus, $\left(1-2 \lambda c_{n}\right)^{-n / 2} \leqq(1-2 \lambda c)^{-n / 2}$ and $\lambda<1 / 2 c$ implies $\lambda<1 / 2 c_{n}$, so we have (2.23). Now, in particular let

$$
y_{k}=X_{k}^{N}-X_{k}=\sum_{|j|<N} a_{j}\left(1-\frac{|j|}{N}\right) \xi_{j+k}-\sum_{j=-\infty}^{\infty} a_{j} \xi_{j+k} .
$$

The spectral density $g(\theta)$ for the process $\left\{Y_{k}\right\}$ is now given by

$$
g(\theta)=\left|\sum_{j=-\infty}^{\infty} a_{j} e^{i j \theta}-\sum_{|j| \leqq N} a_{j}\left(1-\frac{|j|}{N}\right) e^{i j \theta}\right|^{2}=\left|\sqrt{f(\theta)}-h_{N}(\theta)\right|^{2} .
$$

Let $\varepsilon(N)=\sup _{0 \leqq \theta \leqq 2 \pi}|g(\theta)|=\sup _{0 \leqq \theta \leqq 2 \pi}\left|\sqrt{f(\theta)}-h_{N}(\theta)\right|^{2}$. From $(2.3)$ we see $\varepsilon(N)=o(1)$ as $N \rightarrow \infty$, and from (2.23) if $0<\lambda<1 / 2 \varepsilon(N)$, then we have for all $n$,

$$
\frac{1}{n} \log E\left\{\exp \left\{\lambda \sum_{k=1}^{n}\left(x_{k}-x_{k}^{N}\right)^{2}\right\}\right\} \leqq-\frac{1}{2} \log (1-2 \lambda \varepsilon(N)),
$$

which is the lemma.

Lemma 2.5. Let $\omega \in \Omega$ be given by $\omega=\left\{x_{k}\right\},-\infty<k<\infty$ and let $\omega^{\prime} \in \Omega$ be given by $\omega^{\prime}=\left\{x_{k}+\varepsilon_{k}\right\},-\infty<k<\infty$. Then, there is a constant $c$ such that

$$
d\left(\pi_{n}(\omega), \pi_{n}\left(\omega^{\prime}\right)\right) \leqq c \sqrt{\frac{\varepsilon_{1}^{2}+\varepsilon_{2}^{2}+\ldots+\varepsilon_{n}^{2}}{n}} .
$$

Proof. By definition [cf. (2.12)],

$$
d\left(\pi_{n}(\omega), \pi_{n}\left(\omega^{\prime}\right)\right)=\sum_{L=1}^{\infty} \frac{1}{2^{L}} d_{L}\left(\left[\pi_{n}(\omega)\right]_{L}^{1},\left[\pi_{n}\left(\omega^{\prime}\right)\right]_{L}^{1}\right)
$$

Let $\alpha$ and $\beta$ be two $L$-dimensional prob distributions and $\hat{\alpha}$ and $\hat{\beta}$ their characteristic functions. Since uniform convergence of characteristic functions on compact sets in $L$-dimensions is equivalent to weak convergence in $L$-dimensions which is equivalent to convergence in the Prohorov metric $d_{L}$ in $L$-dimensions, we can write

$$
d_{L}(\alpha, \beta)=\sum_{k=1}^{\infty} \frac{1}{2^{k}} \sup _{|\eta| \leqq k}|\hat{\alpha}(\eta)-\hat{\beta}(\eta)| .
$$

In particular, if $\alpha=\delta_{\left(x_{1}, x_{2}, \ldots, x_{L}\right)}$ and $\beta=\delta_{\left(x_{1}+\varepsilon_{1}, \ldots, x_{L}+\varepsilon_{L}\right)}$, we have from (2.26),

$$
\begin{aligned}
d_{L}(\alpha, \beta) & =\sum_{k=1}^{\infty} \frac{1}{2^{k}} \sup _{|\eta| \leqq k}\left|e^{i\langle x, \eta\rangle}-e^{i\langle x+\varepsilon, \eta\rangle}\right| \\
& =\sum_{k=1}^{\infty} \frac{1}{2^{k}} \sup _{|\eta| \leqq k}\left|e^{i\langle\varepsilon, \eta\rangle}-1\right| \\
& \leqq\|\varepsilon\| \sum_{k=1}^{\infty} \frac{k}{2^{k}}=2\|\varepsilon\| .
\end{aligned}
$$


Thus, from the definition of $\pi_{n}$,

$$
\begin{aligned}
& d_{L}\left(\left[\pi_{n}(\omega)\right]_{L}^{1},\left[\pi_{n}\left(\omega^{\prime}\right)\right]_{L}^{1}\right) \\
& \quad \leqq \frac{2}{n}\left(\sqrt{\varepsilon_{1}^{2}+\varepsilon_{2}^{2}+\ldots+\varepsilon_{L}^{2}}+\sqrt{\varepsilon_{2}^{2}+\ldots+\varepsilon_{1+L}^{2}}+\ldots+\sqrt{\varepsilon_{n}^{2}+\ldots+\varepsilon_{n+L-1}^{2}}\right),
\end{aligned}
$$

where $\varepsilon_{n+j}=\varepsilon_{j}$. Using Minkowski's inequality on this last, we get

$$
\begin{aligned}
d_{L}( & {\left.\left[\pi_{n}(\omega)\right]_{L}^{1},\left[\pi_{n}\left(\omega^{\prime}\right)\right]_{L}^{1}\right) } \\
& \leqq 2 \sqrt{\frac{1}{n}\left[\left(\varepsilon_{1}^{2}+\ldots+\varepsilon_{L}^{2}\right)+\left(\varepsilon_{2}^{2}+\ldots+\varepsilon_{2+L}^{2}\right)+\ldots+\left(\varepsilon_{n}^{2}+\ldots+\varepsilon_{n+L}^{2}\right)\right]} \\
& =2 \sqrt{\frac{L}{n}\left(\varepsilon_{1}^{2}+\varepsilon_{2}^{2}+\ldots+\varepsilon_{n}^{2}\right)} .
\end{aligned}
$$

Finally, from (2.25) and this last inequality,

$$
d\left(\pi_{n}(\omega), \pi_{n}\left(\omega^{\prime}\right)\right) \leqq \sum_{L=1}^{\infty} \frac{1}{2^{L}} \cdot 2 \sqrt{\frac{L\left(\varepsilon_{1}^{2}+\ldots+\varepsilon_{n}^{2}\right)}{n}}=c \sqrt{\frac{\varepsilon_{1}^{2}+\ldots+\varepsilon_{n}^{2}}{n}} .
$$

Lemma 2.6. Let $F_{N}$ and $F$ be defined by (2.5) and (2.6) respectively. For any $\delta>0$,

$$
\mu\left\{\omega \in \Omega: d\left(\pi_{n} \cdot F_{N}, \pi_{n} \cdot F\right) \geqq \delta\right\} \leqq \mu\left\{\omega \in \Omega: \sum_{k=1}^{n} \frac{\left(X_{k}^{N}-X_{k}\right)^{2}}{n} \geqq \frac{\delta^{2}}{c^{2}}\right\},
$$

where $c$ is the constant in Lemma 2.5 .

Proof. From (2.1) and (2.4), $X_{k}=\sum_{n=-\infty}^{\infty} a_{n} \xi_{n+k}$ and $x_{k}^{N}=\sum_{|j|<N} a_{j}\left(1-\frac{|j|}{N}\right) \xi_{j+k}$. If we let $\varepsilon_{k}=X_{k}-X_{k}^{N}$, then $X_{k}=X_{k}^{N}+\varepsilon_{k}$. Thus, from Lemma 2.5,

$$
d\left(\pi_{n} \cdot F_{N}, \pi_{n} \cdot F\right) \leqq c \sqrt{\frac{1}{n} \sum_{k=1}^{n}\left(X_{k}-x_{k}^{N}\right)^{2}},
$$

and (2.27) follows.

Lemma 2.7. For every $\delta>0$,

$$
\lim _{N \rightarrow \infty} \varlimsup_{n \rightarrow \infty} \frac{1}{n} \log \mu\left\{\omega \varepsilon \Omega: d\left(\pi_{n} \cdot F_{N}, \pi_{n} \cdot F\right) \geqq \delta\right\}=-\infty .
$$

Proof. For fixed $N$, and $\lambda<1 / 2 \varepsilon(N)$, where $\varepsilon(N)$ is defined above in Lemma 2.4, we have from (2.27) and (2.24) for any $\delta>0$,

$$
\begin{aligned}
& \mu\left\{\omega \in \Omega: d\left(\pi_{n} \cdot F_{N}, \pi_{n} \cdot F\right) \geqq \delta\right\} \leqq \mu\left\{\omega \in \Omega: \frac{1}{n} \sum_{k=1}^{n}\left(X_{k}^{N}-X_{k}\right)^{2} \geqq \frac{\delta^{2}}{c^{2}}\right\} \\
& \leqq E^{\mu}\left\{\exp n \lambda\left(\frac{1}{n} \sum_{k=1}^{n}\left(X_{k}^{N}-X_{k}\right)^{2}\right)\right\} e^{-n \delta^{2} \lambda / c^{2}} \leqq e^{-n \delta^{2} \lambda / c^{2}} e^{-\frac{n}{2} \log (1-2 \lambda \varepsilon(N))} .
\end{aligned}
$$

Therefore, for any $\delta>0$ and $\lambda<1 / 2 \varepsilon(N)$ with $N$ fixed,

$$
\varlimsup_{n \rightarrow \infty} \frac{1}{n} \log \mu\left\{\omega \in \Omega: d\left(\pi_{n} \cdot F_{N}, \pi_{n} \cdot F\right) \geqq \delta\right\} \leqq-\frac{\delta^{2} \lambda}{c^{2}}-\frac{1}{2} \log (1-2 \lambda \varepsilon(N)) .
$$


Since $\varepsilon(N)=o(1)$ as $N \rightarrow \infty$, we get from (2.29) that for all $\delta>0$ and all $\lambda>0$,

$$
\varlimsup_{N \rightarrow \infty} \varlimsup_{n \rightarrow \infty} \frac{1}{n} \log \mu\left\{\omega \in \Omega: d\left(\pi_{n} \cdot F_{N}, \pi_{n} \cdot F\right) \geqq \delta\right\} \leqq-\frac{\delta^{2} \lambda}{c^{2}} .
$$

In this last if we let $\lambda \rightarrow \infty$ we obtain (2.28).

We are now ready to prove (1.5) and (1.6) of the introduction.

Theorem 2.8. If $C \subset \underline{M}_{S}$ is closed, then

$$
\varlimsup_{n \rightarrow \infty} \frac{1}{n} \log Q_{n}(C) \leqq-\inf _{R \in C} H_{f}(R),
$$

and if $G \subset \underline{M}_{S}$ is open, then

$$
\varliminf_{n \rightarrow \infty} \frac{1}{n} \log Q_{n}(G) \geqq-\inf _{R \in G} H_{f}(R),
$$

where $H_{f}(R)$ is defined by (1.9).

Proof. Let $C$ be closed in $\underline{M}_{S}$. Then,

$$
Q_{n}(C)=P\left\{\omega \in \Omega: \pi_{n}(\omega) \in C\right\}=\mu\left\{\omega \in \Omega: \pi_{n} \cdot F(\omega) \in C\right\} .
$$

For any $\delta>0$, we have

$$
\begin{aligned}
Q_{n}(C) & \leqq \mu\left\{\omega \in \Omega: \pi_{n} \cdot F_{n} \in C^{\delta}\right\}+\mu\left\{\omega \in \Omega: d\left(\pi_{n} \cdot F_{N}, \pi_{n} \cdot F\right) \geqq \delta\right\} \\
& =P^{N}\left\{\omega \in \Omega: \pi_{n}(\omega) \in C^{\delta}\right\}+\mu\left\{\omega \in \Omega: d\left(\pi_{n} \cdot F_{N}, \pi_{n} \cdot F\right) \geqq \delta\right\} \\
& =Q_{n}^{N}\left(C^{\delta}\right)+\mu\left\{\omega \in \Omega: d\left(\pi_{n} \cdot F_{N}, \pi_{n} \cdot F\right) \geqq \delta\right\} \\
& \leqq Q_{n}^{N}\left(\bar{C}^{\delta}\right)+\mu\left\{\omega \in \Omega: d\left(\pi_{n} \cdot F_{N}, \pi_{n} \cdot F\right) \geqq \delta\right\} .
\end{aligned}
$$

From Theorem 2.3, this last inequality implies

$$
\varlimsup_{n \rightarrow \infty} \frac{1}{n} \log Q_{n}(C) \leqq \max \left(-\inf _{R \in \bar{C}^{\delta}} H_{f_{N}}(R), A_{N}(\delta)\right),
$$
where $A_{N}(\delta)=\varlimsup_{n \rightarrow \infty} \frac{1}{n} \log \mu\left\{\omega \in \Omega: d\left(\pi_{n} \cdot F_{N}, \pi_{n} \cdot F\right) \geqq \delta\right\}$. From Lemma 2.7 we then
obtain,

$$
\varlimsup_{n \rightarrow \infty} \frac{1}{n} \log Q_{n}(C) \leqq-\varliminf_{N \rightarrow \infty} \inf _{R \in \bar{C}^{\delta}} H_{f_{N}}(R) .
$$

From Lemma 3.10 below it follows that

$$
\underline{\lim _{N \rightarrow \infty}} \inf _{R \in \bar{C}^{\delta}} H_{f_{N}}(R)=\inf _{R \in \bar{C}^{\delta}} H_{f}(R),
$$

where $H_{f}(R)$ is given by (1.9). Using (2.33) in (2.32) we get

$$
\varlimsup_{n \rightarrow \infty} \frac{1}{n} \log Q_{n}(C) \leqq-\inf _{R \in \bar{C}^{\delta}} H_{f}(R) .
$$

Since $C$ is closed and $H_{f}(R)$ is lower semicontinuous (2.34) implies (2.30) on letting $\delta \rightarrow 0$. 
For the lower bound, let $G$ be open in $\underline{M}_{S}$ and let $R$ be any element of $G$. Pick $\delta>0$ and an open set $V$ such that $R \in V \subset V^{\delta} \subset G$. Then

$$
\begin{aligned}
Q_{n}(G) & \geqq Q_{n}\left(V^{\delta}\right)=\mu\left\{\omega \in \Omega: \pi_{n} \cdot F(\omega) \in V^{\delta}\right\} \\
& \geqq \mu\left\{\omega \in \Omega: \pi_{n} \cdot F_{N} \in V\right\}-\mu\left\{\omega \in \Omega: d\left(\pi_{n} \cdot F_{N}, \pi_{n} \cdot F\right) \geqq \delta\right\} \\
& =Q_{n}^{N}(V)-\mu\left\{\omega \in \Omega: d\left(\pi_{n} \cdot F_{N}, \pi_{n} \cdot F\right) \geqq \delta\right\} .
\end{aligned}
$$

Without loss of generality we can assume that $H_{f}(R)<\infty$ for the $R \in G$ we picked.

As before, let

$$
A_{N}(\delta)=\varlimsup_{n \rightarrow \infty} \frac{1}{n} \log \mu\left\{\omega \in \Omega: d\left(\pi_{n} \cdot F_{N}, \pi_{n} \cdot F\right) \geqq \delta\right\} .
$$

From Lemma 2.7 we can choose $N$ so large that $A_{N}(\delta) \leqq-\left(H_{f}(R)+3\right)$, i.e., we can choose $N$ so large that

$$
\mu\left\{\omega \in \Omega: d\left(\pi_{n} \cdot F_{N}, \pi_{n} \cdot F\right) \geqq \delta\right\} \leqq e^{-n\left(H_{f}(R)+3\right)+o(n)} .
$$

Also, since $V$ is open we obtain from Theorem 2.3,

$$
\varliminf_{n \rightarrow \infty} \frac{1}{n} \log Q_{n}^{N}(V) \geqq-\inf _{R^{\prime} \in V} H_{f_{N}}\left(R^{\prime}\right) .
$$

In Lemma 3.9 below we prove that for an $R \in \underline{M}_{S}$ such that $H_{f}(R)<\infty$, there exists a sequence $\left\{R_{N}\right\}$ in $\underline{M}_{S}$ which converges weakly to $R$ and for which $\lim H_{f_{N}}\left(R_{N}\right)=H_{f}(R)$. If we let $0<\eta<1$ be given, then from (2.37) and Lemma 3, since $V$ is a neighborhood of $R$, we can take $N$ so large that

$$
\varliminf_{n \rightarrow \infty} \frac{1}{n} \log Q_{n}^{N}(V) \geqq-\inf _{R^{\prime} \in V} H_{f_{N}}\left(R^{\prime}\right) \geqq-H_{f_{N}}\left(R_{N}\right) \geqq-H_{f}(R)-\eta .
$$

In other words, for $N$ large enough,

$$
Q_{n}^{N}(V) \geqq e^{-n\left(H_{f}(R)+\eta\right)+o(n)} .
$$

Using (2.38) and (2.36) in (2.35) we see that for $N$ large enough

$$
Q_{n}(G) \geqq e^{-n\left(H_{f}(R)+\eta\right)+o(n)}-e^{-n\left(H_{f}(R)+3\right)+o(n)} .
$$

Since $\eta<1$ the first expression on the right is the controlling asymptotic term and we get,

$$
\varliminf_{n \rightarrow \infty} \frac{1}{n} \log Q_{n}(G) \geqq-H_{f}(R)-\eta .
$$

Since $\eta$ is arbitrary and $R$ is an arbitrary element of $G$, we conclude finally that

$$
\varliminf_{n \rightarrow \infty} \frac{1}{n} \log Q_{n}(G) \geqq-\inf _{R \in G} H_{f}(R),
$$

which completes the proof of the theorem. 


\section{Properties of Entropy}

In this section we derive the formula for $H_{f_{N}}(R)$ occurring in Theorem 2.2. We also prove those properties of $H_{f_{N}}(R)$ and $H_{f}(R)$ which we called upon in obtaining the upper and lower bounds in Theorem 2.8.

We will be somewhat more general at first and start with $I(R)$ as defined by (2.16). Note, that for $R \in \underline{M}_{S}$, and with $\phi(y)=\frac{1}{\sqrt{2 \pi}} e^{-y^{2} / 2}$,

$$
\begin{aligned}
I(R) & =E^{R}\left\{\int_{-\infty}^{\infty} r(y \mid \omega) \log \left(\frac{r(y \mid \omega)}{\phi(y)}\right) d y\right\} \\
& =E^{\boldsymbol{R}}\left\{\int_{-\infty}^{\infty} r(y \mid \omega) \log r(y \mid \omega) d y\right\}+\frac{1}{2} \log 2 \pi+\frac{1}{2} E^{R}\left\{\omega^{2}(0)\right\}
\end{aligned}
$$

Let $\left\{a_{k}\right\},-\infty<k<\infty$ be any sequence of reals such that $\sum_{j=1}^{\infty}\left|a_{j}\right|<\infty$ and $|h(\theta)|$ $=\left|\sum_{j=-\infty}^{\infty} a_{j} e^{i j \theta}\right| \neq 0$ for all $\theta \in[0,2 \pi]$. Consider the mapping $\tau: \Omega \rightarrow \Omega$ based on such a sequence $\left\{a_{k}\right\}$ given by $(\tau \omega)(k)=\sum_{j=-\infty}^{\infty} a_{j} \omega(j+k)$. We will call such mappings $\tau$ "admissible."

For $\tau$ such an admissible mapping, define for $R \in \underline{M}_{S}$

$$
H^{\tau}(R)=\inf _{R^{\prime}: R^{\prime} \tau^{-1}=R} I\left(R^{\prime}\right) .
$$

We prove below that if $R$ has spectral measure $G(\theta)$, then

$$
H^{\tau}(R)=I(R)+\frac{1}{4 \pi} \int_{0}^{2 \pi} \frac{d G(\theta)}{|h(\theta)|^{2}}-\frac{1}{4 \pi} \int_{0}^{2 \pi} d G(\theta)+\frac{1}{2 \pi} \int_{0}^{2 \pi} \log \mid h(\theta) d \theta
$$

Before proving (3.3) we need to examine how $I\left(R \tau^{-1}\right)$ is related to $I(R)$ for an admissible map $\tau$. We start with some preliminary lemmas. The first is easy and we omit the proof. The second is a classic result found in standard references on matrices.

Lemma 3.1. Let $R \in \underline{M}_{S}$ and let $R_{n}\left(y_{1}, y_{2}, \ldots, y_{n}\right)$ be the $n$-dimensional joint density for the $R$ process, i.e., $R_{n}\left(y_{1}, y_{2}, \ldots, y_{n}\right) d y_{1} \ldots d y_{n}=R\left\{X_{1} \in d y_{1}, \ldots, X_{n} \in d y_{n}\right\}$. Define, with $\phi(y)=\frac{1}{\sqrt{2 \pi}} e^{-y^{2} / 2}$,

$$
I_{n}(R)=\int_{-\infty}^{\infty} \ldots R_{n}\left(y_{1}, y_{2}, \ldots, y_{n}\right) \log \left(\frac{R_{n}\left(y_{1}, y_{2}, \ldots, y_{n}\right)}{\prod_{j=1}^{n} \phi\left(y_{j}\right)}\right) d y_{1} \ldots d y_{n}
$$


Then, $I_{n}(R)-I_{n-1}(R)$ is monotone increasing in $n, \lim _{n \rightarrow \infty}\left(I_{n}(R)-I_{n-1}(R)\right)=I(R)$ so that also

$$
\lim _{n \rightarrow \infty} \frac{I_{n}(R)}{n}=I(R) \text {. }
$$

Lemma 3.2. Let $D$ be the determinant of a circulant matrix of order $n$, i.e.,

$$
D=\left|\begin{array}{ccccc}
d_{1} & d_{2} & \ldots & d_{n} \\
d_{n} & d_{1} & d_{2} & \ldots & d_{n-1} \\
\vdots & & & \\
d_{2} & d_{3} & \ldots & d_{n} & d_{1}
\end{array}\right| .
$$

Then

$$
D=\prod_{\xi \in Z_{n}}\left(d_{1}+d_{2} \xi+\ldots+d_{n} \xi^{n-1}\right),
$$

where $Z_{n}$ is the set of $n^{\text {th }}$ roots of unity.

Lemma 3.3. Let $f(x)$ be a probability density in d-dimensions and let $g(x)$ be the d-dimensional Gaussian density, $g(x)=\frac{1}{(2 \pi)^{d / 2}} e^{-(1 / 2)\|x\|^{2}}$. Let $A$ be a nonsingular
matrix. Then,

$$
\begin{aligned}
& \int_{\boldsymbol{R}^{d}}\left(\frac{f\left(A^{-1} x\right)}{|A|}\right) \log \left(\frac{f\left(A^{-1} x\right) /|A|}{g(x)}\right) d x=\int_{\boldsymbol{R}^{d}} f(y) \log \frac{f(y)}{g(y)} d y \\
& \quad+\frac{1}{2} \int_{R^{d}}\left(\|A y\|^{2}-\|y\|^{2}\right) f(y) d y-\log |A| .
\end{aligned}
$$
Proof. We observe that if $\alpha(d x)=f(x) d x$, then $\left(\alpha A^{-1}\right)(d x)=\frac{f\left(A^{-1} x\right)}{|A|} d x$. Now,
letting $x=A y$,

$$
\begin{aligned}
& \int_{R^{d}}\left(\frac{f\left(A^{-1} x\right)}{|A|}\right) \log \left(\frac{f\left(A^{-1} x\right) /|A|}{g(x)}\right) d x=\int_{R^{d}} f(y) \log \left(\frac{f(y)}{g(A y)}\right) d x-\log |A| \\
& \quad=\int_{R^{d}} f(y) \log \left(\frac{f(y)}{g(y)}\right) d y+\int_{R^{d}} f(y) \log \left(\frac{g(y)}{g(A y)}\right) d y-\log |A| \\
& \quad=\int_{R^{d}} f(y) \log \frac{f(y)}{g(y)} d y+\frac{1}{2} \int_{R^{d}}\left(\|A y\|^{2}-\|y\|^{2}\right) f(y) d y-\log |A| .
\end{aligned}
$$

As a preparatory lemma we consider admissible maps of a special kind - where only a finite number of the elements in $\left\{a_{k}\right\}$ are different from 0 .

Lemma 3.4. For any $R \in \underline{M}_{S}$, let $I(R)$ be defined by (3.1) and let $\tau: \Omega \rightarrow \Omega$ be defined by $(\tau \omega)(j)=\sum_{k=-\infty}^{\infty} a_{k} \omega(k+j)$, where, for some $K, a_{k}=0$ if $|k|>K$ and $|h(\theta)|$ $=\sum_{k=-\infty}^{\infty} a_{k} e^{i k \theta} \| \neq 0$ for all $\theta \in[0,2 \pi]$. Then,

$$
I\left(R \tau^{-1}\right) \leqq I(R)+\frac{1}{2}\left[E^{R \tau^{-1}}\left\{\omega^{2}(0)\right\}-E^{R}\left\{\omega^{2}(0)\right\}-\frac{1}{2 \pi} \int_{0}^{2 \pi} \log |h(\theta)| d \theta\right.
$$


Proof. Let $n>K$ where $K$ is such that $a_{k}=0$ if $|k|>K$. Consider the $(2 n+1)$ $\times(2 n+1)$ circulant matrix

$$
A_{n+1}=\left|\begin{array}{lllc}
a_{0} & a_{1} \ldots a_{n} & a_{-n} \ldots a_{-1} \\
a_{-1} & a_{0} \ldots a_{n-1} & \ldots a_{-2} \\
a_{1} & a_{2} & a_{-n} & a_{0}
\end{array}\right| .
$$

Let $E_{2 n+1}$ be $(2 n+1)$ dimensional space and define a mapping $E_{2 n+1} \rightarrow E_{2 n+1}$ by $x \rightarrow A_{2 n+1} x$. If $\omega \in \Omega$, let $\Gamma_{2 n+1} \omega=(\omega(j))_{|j| \leqq n}$, i.e., if $\omega=\left\{x_{k}\right\},-\infty<k<\infty$, then $\Gamma_{2 n+1}$ is the section:

$$
x_{-n}, x_{-n+1}, \ldots, x_{-1}, x_{0}, x_{1}, \ldots, x_{n-1}, x_{n} .
$$

It is easy to see that if $|i| \leqq n-K$, then

$$
(\tau \omega)(i)=\left(A_{2 n+1} \Gamma_{2 n+1} \omega\right)(i) .
$$

Define the density $R_{2 n+1}^{\prime}\left(y_{-n}, \ldots, y_{0}, \ldots, y_{n}\right)$ by

$$
R_{2 n+1}^{\prime}\left(y_{-n}, \ldots, y_{0}, \ldots, y_{n}\right) d y_{-n} \ldots d y_{0} \ldots d y_{n}=R \tau^{-1} \Gamma_{2 n+1}^{-1},
$$

and similarly define the density $\hat{R}_{2 n+1}^{\prime}$ by

$$
\hat{R}_{2 n+1}^{\prime}\left(y_{-n}, \ldots, y_{0}, \ldots, y_{n}\right) d y_{-n}, \ldots, d y_{0} \ldots d y_{n}=R \Gamma_{2 n+1}^{-1} A_{2 n+1}^{-1} \text {. }
$$

From the definition of $I_{n}(R)$ in Lemma 3.1,

$$
\begin{aligned}
I_{2 k+1}\left(R \tau^{-1}\right)= & \int_{-\infty}^{\infty} \ldots R_{2 k+1}^{\prime}\left(y_{-k}, \ldots, y_{0}, \ldots, y_{k}\right) \\
& \cdot \log \left(\frac{R_{2 k+1}^{\prime}\left(y_{-k}, \ldots, y_{0}, \ldots, y_{k}\right)}{\left(\frac{1}{\sqrt{2 \pi}}\right)^{2 k+1} \exp \left\{-1 \frac{1}{2} \sum_{j=-k}^{k} y_{j}^{2}\right\}}\right) d y_{-k} d y_{0} \ldots d y_{k} .
\end{aligned}
$$

From (3.7) we see that $R \tau^{-1} \Gamma_{2 n+1}^{-1}=R \Gamma_{2 l+1}^{-1} A_{2 l+1}^{-1} \Gamma_{2 n+1}^{-1}$ provided $l \geqq n+K$. In particular, if we choose $n=k, l=k+K$, we have

$$
R \tau^{-1} \Gamma_{2 k+1}^{-1}=R \Gamma_{2(k+K)+1}^{-1} A_{2(k+K)+1}^{-1} \Gamma_{2 k+1}^{-1} .
$$

Therefore, from (3.8) we obtain

$$
\begin{aligned}
I_{2 k+1}\left(R \tau^{-1}\right) \leqq & \int_{-\infty}^{\infty} \ldots \hat{R}_{2(k+K)+1}^{\prime}\left(y_{-k-k}, \ldots, y_{0}, \ldots, y_{k+K}\right) \\
& \cdot \log \frac{\hat{R}_{2(k+K)+1}^{\prime}\left(y_{-K+k}, \ldots, y_{0}, \ldots, y_{k+K}\right)}{\left(\frac{1}{\sqrt{2 \pi}}\right)^{2(k+K)+1} \exp \left\{-\frac{1}{2} \sum_{j=-k-K}^{k+K} y_{j}^{2}\right\}} d y_{-k-K} \ldots d y_{k+K}
\end{aligned}
$$

From Lemma 3.3 and (3.9) we get the estimate

$$
\begin{aligned}
I_{2 k+1}\left(R \tau^{-1}\right) \leqq & I_{2(k+K)+1}(R)-\log \left|A_{2(k+K)+1}\right| \\
& +\frac{1}{2} E^{R}\left\{\left\|\mathrm{~A}_{2(k+K)+1} \Gamma_{2(k+K)+1} \omega\right\|^{2}-\left\|\Gamma_{2(k+K)+1} \omega\right\|^{2}\right\} .
\end{aligned}
$$


Since $R$ is stationary and $a_{k}=0$ if $|k|>K$,

$$
\lim _{n \rightarrow \infty} \frac{1}{2 n+1} E^{R}\left\{\left\|A_{2 n+1} \Gamma_{2 n+1} \omega\right\|^{2}\right\}=E^{R}\left\{\left|\sum_{j=-\infty}^{\infty} a_{j} \omega(j)\right|^{2}\right\}=E^{R \tau^{-1}}\left\{\omega^{2}(0)\right\},
$$

and

$$
\lim _{n \rightarrow \infty} \frac{1}{2 n+1} E^{R}\left\{\left\|\Gamma_{2 n+1} \omega\right\|^{2}\right\}=E^{R}\left\{\omega^{2}(0)\right\}
$$

Let $c=\lim _{n \rightarrow \infty} \frac{1}{2 n+1} \log \left|A_{2 n+1}\right|$, divide both sides of (3.10) by $2 k+1$, let $k \rightarrow \infty$, and use Lemma 3.1 to obtain

$$
I\left(R \tau^{-1}\right) \leqq I(R)-c+\frac{1}{2}\left[E^{R \tau^{-1}}\left\{\omega^{2}(0)\right\}-E^{R}\left\{\omega^{2}(0)\right\}\right] .
$$

Thus, it remains to show that

$$
c=\frac{1}{2 \pi} \int_{0}^{2 \pi} \log |h(\theta)| d \theta,
$$

where $h(\theta)=\sum_{k=-\infty}^{\infty} a_{k} e^{i k \theta}$. Since $A_{2 n+1}$ is a circulant matrix, Lemma 3.2 applies. We note that if $\xi \in Z_{2 n+1}$ then $\xi^{-j}=\xi^{2 n+1-j}$. Since $a_{k}=0$ if $|k|>K$, we get

$$
\begin{aligned}
& \lim _{n \rightarrow \infty} \frac{1}{2 n+1} \log \left|A_{2 n+1}\right|=\lim _{n \rightarrow \infty} \frac{1}{2 n+1} \log \prod_{\xi \in Z_{2 n+1}}\left(\sum_{|j| \leqq n} a_{j} \xi^{j}\right) \\
& \quad=\lim _{n \rightarrow \infty} \frac{1}{2 n+1} \sum_{\xi \in Z_{2 n+1}} \log \sum_{j=-\infty}^{\infty} a_{j} \xi^{j}=\frac{1}{2 \pi} \int_{0}^{2 \pi} \log \left|\sum_{j=-\infty}^{\infty} a_{j} e^{i j \theta}\right| d \theta,
\end{aligned}
$$

where, of course, in the last equality we used the properties of roots of unity and that the last expression is finite since $|h(\theta)| \neq 0$ for all $\theta \in[0,2 \pi]$ by hypothesis.

In the preceding lemma we showed inequality (3.6) under the hypothesis that the admissible mapping $\tau$ was in fact based on coefficients $\left\{a_{k}\right\}$ only a finite number of which were different from zero. Using that result we can now show that for any admissible mapping equality actually holds in (3.6).

Lemma 3.5. Let $\tau$ be any admissible mapping. Then, for any $R \in \underline{M}_{S}$,

$I\left(R \tau^{-1}\right)=I(R)+\frac{1}{2}\left[E^{R \tau^{-1}}\left\{\omega^{2}(0)\right\}-E^{R}\left\{\omega^{2}(0)\right\}\right]-\frac{1}{2 \pi} \int_{0}^{2 \pi} \log \left|\sum_{k=-\infty}^{\infty} a_{k} e^{i k \theta}\right| d \theta$.

Proof. First define the map $\tau_{n}$ by $\left(\tau_{n} \omega\right)(j)=\sum_{k=-n}^{n} a_{k} \omega(k+j)$ with the same $a_{k}$ 's as in the statement of the lemma. We apply Lemma 3.4 to $\tau_{n}$ so that for any $R \in \underline{M}_{S}$,

$$
\begin{aligned}
I\left(R \tau_{n}^{-1}\right) \leqq & I(R)+\frac{1}{2}\left[E^{R \tau_{n}^{-1}}\left\{\omega^{2}(0)\right\}-E^{R}\left\{\omega^{2}(0)\right\}\right] \\
& -\frac{1}{2 \pi} \int_{0}^{2 \pi} \log \left|\sum_{k=-n}^{n} a_{k} e^{i k \theta}\right| d \theta .
\end{aligned}
$$


Since $I(R)$ is lower semicontinuous on $\underline{M}_{S}, I\left(R \tau^{-1}\right) \leqq \underline{\lim _{n \rightarrow \infty}} I\left(R \tau_{n}^{-1}\right)$. Moreover, $\lim _{n \rightarrow \infty} E^{R \tau_{n}^{-1}}\left\{\omega^{2}(0)\right\}=E^{R \tau^{-1}}\left\{\omega^{2}(0)\right\}$, and

$$
\lim _{n \rightarrow \infty} \frac{1}{2 \pi} \int_{0}^{2 \pi} \log \left|\sum_{k=-n}^{n} a_{k} e^{i k \theta}\right| d \theta=\frac{1}{2 \pi} \int_{0}^{2 \pi} \log \left|\sum_{k=-\infty}^{\infty} a_{k} e^{i k \theta}\right| d \theta
$$

since $\left|\sum_{k=-\infty}^{\infty} a_{k} e^{i k \theta}\right| \neq 0$ for all $\theta \in[0,2 \pi]$. Thus, from (3.13) we conclude, for $R \in \underline{M}_{S}$,

$$
\begin{aligned}
I\left(R \tau^{-1}\right) \leqq & I(R)+\frac{1}{2}\left[E^{R \tau^{-1}}\left\{\omega^{2}(0)\right\}-E^{R}\left\{\omega^{2}(0)\right\}\right] \\
& -\frac{1}{2 \pi} \int_{0}^{2 \pi} \log \left|\sum_{k=-\infty}^{\infty} a_{k} e^{i k \theta}\right| d \theta
\end{aligned}
$$

for any admissible mapping $\tau$. Let $g(\theta)=\sum_{j=-\infty}^{\infty} a_{j} e^{i j \theta}$. By our hypothesis this is an absolutely convergent Fourier series and $g(\theta) \neq 0$ for any $\theta \in[0,2 \pi]$. Wiener's theorem says that the $L^{2}$-function $1 / g(\theta)$ has a Fourier series $\frac{1}{g(\theta)}=\sum_{j=-\infty}^{\infty} b_{j} e^{i j \theta}$ and $\sum_{j=-\infty}^{\infty}\left|b_{j}\right|<\infty$. Of course, $\left|\sum_{j=-\infty}^{\infty} b_{j} e^{i j \theta}\right| \neq 0$ for all $\theta \in[0,2 \pi]$.

Consider then the admissible map $\sigma$ given by $(\sigma \omega)(j)=\sum_{k=-\infty}^{\infty} b_{k} \omega(k+j)$ and note that $\sigma=\tau^{-1}$. Apply (3.14) to $\sigma$ and we get

$$
\begin{aligned}
I\left(R \sigma^{-1}\right) \leqq & I(R)+\frac{1}{2}\left[E^{R \sigma^{-1}}\left\{\omega^{2}(0)\right\}-E^{R}\left\{\omega^{2}(0)\right\}\right] \\
& +\frac{1}{2 \pi} \int_{0}^{2 \pi} \log \left|\sum_{k=-\infty}^{\infty} a_{k} e^{i k \theta}\right| d \theta
\end{aligned}
$$

where the sign in the last term on the right is now positive since $\left|\sum_{k=-\infty}^{\infty} a_{k} e^{i k \theta}\right|$ $=\frac{1}{\left|\sum_{k=-\infty}^{\infty} b_{k} e^{i k \theta}\right|}$. But (3.15) is true for all $R \in \underline{M}_{S}$ and, therefore, true in particular if we replace $R$ in (3.15) by $R \tau^{-1}$ yielding, since $\sigma=\tau^{-1}$,

or

$$
\begin{aligned}
I\left(R \tau^{-1} \sigma^{-1}\right)= & I(R) \leqq I\left(R \tau^{-1}\right)+\frac{1}{2}\left[E^{R}\left\{\omega^{2}(0)\right\}-E^{R \tau^{-1}}\left\{\omega^{2}(0)\right\}\right] \\
& +\frac{1}{2 \pi} \int_{0}^{2 \pi} \log \left|\sum_{k=-\infty}^{\infty} a_{k} e^{i k \theta}\right| d \theta
\end{aligned}
$$

$$
\begin{gathered}
I\left(R \tau^{-1}\right) \geqq I(R)+\frac{1}{2}\left[E^{R \tau^{-1}}\left\{\omega^{2}(0)\right\}-E^{R}\left\{\omega^{2}(0)\right\}\right] \\
-\frac{1}{2 \pi} \int_{0}^{2 \pi} \log \left|\sum_{k=-\infty}^{\infty} a_{k} e^{i k \theta}\right| d \theta
\end{gathered}
$$


Inequalities (3.14) and (3.16) give us (3.12) for any admissible map $\tau$ which is the lemma.

From the preceding lemma we see that for any admissible mapping $\tau, I(R)<\infty$ if and only if $I\left(R \tau^{-1}\right)<\infty$. Since an admissible mapping $\tau$ is $1-1$ we observe that for any $\tau$ in this class, and with $\sigma=\tau^{-1}$,

$$
\begin{aligned}
H^{\tau}(R) & =\inf _{R^{\prime}: R^{\prime} \tau^{-1}=R} I\left(R^{\prime}\right)=I\left(R \sigma^{-1}\right) \\
& =I(R)+\frac{1}{2}\left[E^{R \sigma^{-1}}\left\{\omega^{2}(0)\right\}-E^{R}\left\{\omega^{2}(0)\right\}\right]+\frac{1}{2 \pi} \int_{0}^{2 \pi} \log |h(\theta)| d \theta,
\end{aligned}
$$

where $h(\theta)=\sum_{j=-\infty}^{\infty} a_{j} e^{i j \theta}$, and $\tau$ is given by $(\tau \omega)(j)=\sum_{k=-\infty}^{\infty} a_{k} \omega(k+j)$. Note, that in (3.17) the sign before the last term on the right is + [cf.(3.12)] because $\sigma=\tau^{-1}$ and the sequence $\left\{a_{k}\right\}$ belongs to $\tau$.

Moreover if $R \in \underline{M}_{S}$ has spectral measure $G(\theta)$, then $\frac{1}{2 \pi} \int_{0}^{2 \pi} d G(\theta)=E^{R}\left\{\omega^{2}(0)\right\}$ and also since $\sigma=\tau^{-1}$ we have $E^{R \sigma^{-1}}\left\{\omega^{2}(0)\right\}=\frac{1}{2 \pi} \int_{0}^{2 \pi} \frac{d G(\theta)}{|h(\theta)|^{2}}$. Hence, we can rewrite (3.17) in the form

$$
\begin{aligned}
H^{\tau}(R)= & I(R)+\frac{1}{4 \pi} \int_{0}^{2 \pi} \frac{d G(\theta)}{|h(\theta)|^{2}} \\
& -\frac{1}{4 \pi} \int_{0}^{2 \pi} d G(\theta)+\frac{1}{2 \pi} \int_{0}^{2 \pi} \log |h(\theta)| d \theta,
\end{aligned}
$$

which is formula (3.3) at the beginning of this section.

In formula (3.18) which holds for any admissible $\tau$, replace $I(R)$ by the last line in (3.1), using again that $E^{R}\left\{w^{2}(0)\right\}=\frac{1}{2 \pi} \int_{0}^{2 \pi} d G(\theta)$, so

$$
\begin{aligned}
H^{\tau}(R)= & E^{R}\left\{\int_{-\infty}^{\infty} r(y \mid \omega) \log r(y \mid \omega) d y\right\} \\
& +\frac{1}{2} \log 2 \pi+\frac{1}{4 \pi} \int_{0}^{2 \pi} \frac{d G(\theta)}{|h(\theta)|^{2}}+\frac{1}{2 \pi} \int_{0}^{2 \pi} \log |h(\theta)| d \theta .
\end{aligned}
$$

Now, in particular, consider the mapping $F_{N}: \Omega \rightarrow \Omega$ given by (2.5) or more precisely, its induced map $\widetilde{F}_{N}$ in $\underline{M}_{S}$. This is an admissible map since

$$
\left(F_{N} \omega\right)(j)=\sum_{|k|<N} a_{k}\left(1-\frac{|k|}{N}\right) \omega(k+j),
$$

and therefore at most a finite number of the coefficients defining this map are different from 0. Moreover, $h_{N}(\theta)=\sum_{|k|<N} a_{k}\left(1-\frac{|k|}{N}\right) e^{i k \theta}$, defined in (2.2), never vanishes since by Fejer's theorem,

$$
h_{N}(\theta)=\int_{0}^{2 \pi} \sqrt{f(\phi)} \psi_{N}(\theta-\phi) d \phi,
$$


where

$$
\psi_{N}(\theta)=\sum_{|k|<N}\left(1-\frac{|k|}{N}\right) e^{i k \theta}
$$

is strictly positive. If as before we let $f_{N}(\theta)=\left|h_{N}(\theta)\right|^{2}$, then $f_{N}(\theta)$ is the spectral density of the process $\left\{X_{k}^{N}\right\}$, and so with $\tau=F_{N}$ and $H_{f_{N}}(R)=H^{\tau}(R)$, we get from (3.19)

$$
\begin{aligned}
H_{f_{N}}(R)= & E^{R}\left\{\int_{-\infty}^{\infty} r(y \mid \omega) \log r(y \mid \omega) d y\right\}+\frac{1}{2} \log 2 \pi \\
& +\frac{1}{4 \pi} \int_{0}^{2 \pi} \frac{d G(\theta)}{f_{N}(\theta)}+\frac{1}{4 \pi} \int_{0}^{2 \pi} \log f_{N}(\theta) d \theta
\end{aligned}
$$

Formula (3.20) is the same as in Sect. 2 just after (2.17).

Finally, we must see in what sense $H_{f_{N}}(R)$ approximates $H_{f}(R)$ as defined by formula (1.9). We begin with the following well known lemma.

Lemma 3.6. Let $\phi(x)$ be a probability density on $\mathbb{R}$ with two moments and let $\sigma^{2}(\phi)$ be the variance of $\phi$. Then, for any $\alpha>0$,

$$
\int_{-\infty}^{\infty} \phi(y) \log \phi(y) d y \geqq-\frac{1}{2} \log 2 \pi \alpha-\frac{1}{2 \alpha} \sigma^{2}(\phi) .
$$

Proof. Let $\phi_{\sigma, \mu}=\frac{1}{\sigma \sqrt{2 \pi}} \exp \left\{-\frac{(y-\mu)^{2}}{2 \sigma^{2}}\right\}$. We note

$$
\int_{-\infty}^{\infty} \phi(y) \log \phi(y) d y=\int_{-\infty}^{\infty} \phi(y) \log \left(\frac{\phi(y)}{\phi_{\sigma, \mu}(y)}\right) d y+\int_{-\infty}^{\infty} \phi(y) \log \phi_{\sigma, \mu}(y) d y .
$$

The first term on the right is nonnegative being the entropy of one probability density with respect to another. Now, $\log \phi_{\sigma, \mu}(y)=-\log (\sigma \sqrt{2 \pi})-\frac{1}{2 \sigma^{2}}(y-\mu)^{2}$, and therefore

$$
\int_{-\infty}^{\infty} \phi(y) \log \phi(y) d y \geqq-\frac{1}{2} \log \left(2 \pi \sigma^{2}\right)-\frac{1}{2 \sigma^{2}} \int_{-\infty}^{\infty}(y-\mu)^{2} \phi(y) d y .
$$

If, in particular, we choose $\mu=\int_{-\infty}^{\infty} y \phi(y) d y$ and $\sigma^{2}=\alpha$ we obtain (3.21).

Lemma 3.7. Let $\tau$ be an admissible map and for $R \in \underline{M}_{S}$ let

$$
e(R)=\int_{-\infty}^{\infty} r(y \mid \omega) \log r(y \mid \omega) d y .
$$

If $R^{\prime}=R \tau^{-1}$, then

$$
e\left(R^{\prime}\right)=e(R)-\frac{1}{2 \pi} \int_{0}^{2 \pi} \log |h(\theta)| d \theta
$$


where $h(\theta)=\sum_{j=-\infty}^{\infty} a_{j} e^{i j \theta}$ and $\left\{a_{j}\right\},-\infty<j<\infty$ are the coefficients defining the map $\tau$.

Proof. From (3.12) and (3.1)

$$
\begin{aligned}
I\left(R^{\prime}\right) & =I(R)+\frac{1}{2}\left[E^{R^{\prime}}\left\{\omega^{2}(0)\right\}-E^{R}\left\{\omega^{2}(0)\right\}\right]-\frac{1}{2 \pi} \int_{0}^{2 \pi} \log |h(\theta)| d \theta \\
& =e(R)+\frac{1}{2} \log 2 \pi+\frac{1}{2} E^{R^{\prime}}\left\{\omega^{2}(0)\right\}-\frac{1}{2 \pi} \int_{0}^{2 \pi} \log |h(\theta)| d \theta
\end{aligned}
$$

Using (3.1) on the left of this last equation we see that

$$
\begin{aligned}
& e\left(R^{\prime}\right)+\frac{1}{2} \log 2 \pi+\frac{1}{2} E^{R^{\prime}}\left\{\omega^{2}(0)\right\} \\
& \quad=e(R)+\frac{1}{2} \log 2 \pi+\frac{1}{2} E^{R^{\prime}}\left\{\omega^{2}(0)\right\}-\frac{1}{2 \pi} \int_{0}^{2 \pi} \log |h(\theta)| d \theta,
\end{aligned}
$$

from which (3.22) follows.

Lemma 3.8. With $f_{N}(\theta)$ and $f(\theta)$ as in Sect. 2,

$$
\lim _{N \rightarrow \infty} \int_{0}^{2 \pi} \log f_{N}(\theta) d \theta=\int_{0}^{2 \pi} \log f(\theta) d \theta .
$$

Proof. It suffices to show, since $f_{N}(\theta)=h_{N}^{2}(\theta)$, that

$$
\lim _{N \rightarrow \infty} \int_{0}^{2 \pi} \log h_{N}(\theta) d \theta=\int_{0}^{2 \pi} \log \sqrt{f(\theta)} d \theta .
$$

Now, $h_{N}(\theta)=\sqrt{f} * \psi_{N}$, where $\psi_{N}$ is Fejer's kernel. Therefore, by Jensen's inequality,

$$
\log h_{N}(\theta)=\log \left(\sqrt{f} * \psi_{N}\right) \geqq \log \sqrt{f} * \psi_{N},
$$

so that

$$
\int_{0}^{2 \pi} \log h_{N}(\theta) d \theta \geqq \int_{0}^{2 \pi}\left(\log \sqrt{f} * \psi_{N}\right) d \theta=\int_{0}^{2 \pi} \log \sqrt{f(\theta)} d \theta,
$$

and hence

$$
\lim _{N \rightarrow \infty} \int_{0}^{2 \pi} \log h_{N}(\theta) d \theta \geqq \int_{0}^{2 \pi} \log \sqrt{f(\theta)} d \theta
$$

On the other hand, from (2.3) we have that $\left\{h_{N}(\theta)\right\}$ are uniformly bounded and $\log h_{N}(\theta) \rightarrow \log \sqrt{f}$, so that from Fatou's lemma

$$
\varlimsup_{N \rightarrow \infty} \int_{0}^{2 \pi} \log h_{N}(\theta) d \theta \leqq \int_{0}^{2 \pi} \log \sqrt{f(\theta)} d \theta
$$


Lemma 3.9. Let $R \in \underline{M}_{S}$ such that $H_{f}(R)<\infty$; then there exists a sequence $\left\{R_{N}\right\} \in \underline{M}_{S}$ such that $\left\{R_{N}\right\}$ converges weakly to $R$ and

$$
\lim _{N \rightarrow \infty} H_{f_{N}}\left(R_{N}\right)=H_{f}(R) \text {. }
$$

Proof. From its definition (1.9),

$$
H_{f}(R)=e(R)+\frac{1}{2} \log 2 \pi+\frac{1}{4 \pi} \int_{0}^{2 \pi} \frac{d G(\theta)}{f(\theta)}+\frac{1}{4 \pi} \int_{0}^{2 \pi} \log f(\theta) d \theta,
$$

where $G(\theta)$ is the spectral measure of $R$ and $e(R)$ is as defined in Lemma 3.7. From (3.20), for any $R^{\prime} \in \underline{M}_{S}$,

$$
H_{f_{N}}\left(R^{\prime}\right)=e\left(R^{\prime}\right)+\frac{1}{2} \log 2 \pi+\frac{1}{4 \pi} \int_{0}^{2 \pi} \frac{d G^{\prime}(\theta)}{f_{N}(\theta)}+\frac{1}{4 \pi} \int_{0}^{2 \pi} \log f_{N}(\theta) d \theta
$$

where $G^{\prime}(\theta)$ is the spectral measure of $R^{\prime}$. The way we construct the desired sequence $\left\{R_{N}\right\}$ is to define $R_{N}=R \tau_{N}^{-1}$, where $\left\{\tau_{N}\right\}$ is a suitably chosen sequence of admissible mappings which first of all will approach the identity map as $N \rightarrow \infty$, so that $R_{N}$ will converge weaky to $R$. Secondly, $\left\{\tau_{N}\right\}$ will have to be selected so that (3.24) holds. Choosing $\tau_{N}$ means choosing a suitable sequence of coefficients. Let $g_{N}(\theta)=\sum_{j=-\infty}^{\infty} c_{k}^{(N)} e^{i k \theta}$, where $\left\{c_{k}^{(N)}\right\}$ are the defining coefficients of the admissible $\operatorname{map} \tau_{N}$. Taking into account Lemma 3.7 and 3.8, we see that for $\tau_{N}$ to satisfy the necessary requirements, $g_{N}(\theta)$ must satisfy:

$$
\begin{gathered}
\lim _{N \rightarrow \infty} \int_{0}^{2 \pi} \log \left|g_{N}(\theta)\right| d \theta=0, \\
\lim _{N \rightarrow \infty} \int_{0}^{2 \pi} \frac{\left|g_{N}(\theta)\right|^{2}}{f_{N}(\theta)} d G(\theta)=\int_{0}^{2 \pi} \frac{d G(\theta)}{f(\theta)}, \\
\lim _{N \rightarrow \infty} \int_{0}^{2 \pi}\left|g_{N}(\theta)-1\right|^{2} d G(\theta)=0 .
\end{gathered}
$$

Condition (1), because of (3.22), guarantees $e\left(R_{N}\right) \rightarrow e(R)$. Since the spectral measure of $R_{N}$, call it $d G_{N}(\theta)=\left|g_{N}(\theta)\right|^{2} d G(\theta)$, we see that (2), (1) and Lemma 3.8 insure that $H_{f_{N}}\left(R_{N}\right) \rightarrow H_{f}(R)$. Condition (3) insures that $\tau_{N}$ approaches the identity because it is the same as requiring $E^{R}\left\{\left|\omega(0)-\left(\tau_{N} \omega\right)(0)\right|^{2}\right\} \rightarrow 0$ as $N \rightarrow \infty$.

Let $g_{N, \varepsilon}(\theta)=\frac{\sqrt{f_{N}(\theta)}}{\sqrt{f_{N}(\theta)+\varepsilon}}$. We will show

$$
\begin{gathered}
\lim _{\varepsilon \rightarrow 0} \lim _{N \rightarrow \infty}\left|\int_{0}^{2 \pi} \log \right| g_{N, \varepsilon}(\theta)|d \theta|=0, \\
\lim _{\varepsilon \rightarrow 0} \lim _{N \rightarrow \infty}\left|\int_{0}^{2 \pi} \frac{\left|g_{N, \varepsilon}(\theta)\right|^{2} d G(\theta)}{f_{N}(\theta)}-\int_{0}^{2 \pi} \frac{d G(\theta)}{f(\theta)}\right|=0,
\end{gathered}
$$

and

$$
\lim _{\varepsilon \rightarrow 0} \lim _{N \rightarrow \infty} \int_{0}^{2 \pi}\left|g_{N, \varepsilon}(\theta)-1\right|^{2} d G(\theta)=0
$$


This will mean there exists a sequence $\varepsilon_{N} \rightarrow 0$ as $N \rightarrow \infty$ such that if we define $g_{N}(\theta)$ $=g_{N, \varepsilon_{N}}(\theta)$, then $(1)^{\prime},(2)^{\prime}$, and (3)' imply (1), (2), (3) and hence the lemma. Because of (2.3), $\quad g_{N, \varepsilon}(\theta) \rightarrow \sqrt{f(\theta)} /(\sqrt{f(\theta)}+\varepsilon)$ uniformly for $\theta \in[0,2 \pi]$. Also $\frac{\left|g_{N, \varepsilon}(\theta)\right|^{2}}{f_{N}(\theta)}$ $\rightarrow \frac{1}{(\sqrt{f(\theta)}+\varepsilon)^{2}}$ uniformly for $\theta \in[0,2 \pi]$. Thus,

$$
\begin{gathered}
\lim _{N \rightarrow \infty} \int_{0}^{2 \pi} \log \mid g_{N, \varepsilon}(\theta) d \theta=\int_{0}^{2 \pi} \log \left(\frac{\sqrt{f(\theta)}}{\sqrt{f(\theta)}+\varepsilon}\right) d \theta, \\
\lim _{N \rightarrow \infty} \int_{0}^{2 \pi} \frac{\left|g_{N, \varepsilon}(\theta)\right|^{2} d G(\theta)}{f_{N}(\theta)}=\int_{0}^{2 \pi} \frac{d G(\theta)}{(\sqrt{f(\theta)}+\varepsilon)^{2}},
\end{gathered}
$$

and

$$
\lim _{N \rightarrow \infty} \int_{0}^{2 \pi}\left|g_{N, \varepsilon}(\theta)-1\right|^{2} d G(\theta)=\int_{0}^{2 \pi}\left|\frac{\sqrt{f(\theta)}}{\sqrt{f(\theta)}+\varepsilon}-1\right|^{2} d G(\theta) .
$$

In (3.27), since by hypothesis $\int_{0}^{2 \pi} \log f(\theta) d \theta>-\infty$, we see $f(\theta)>0$ almost everywhere on $[0,2 \pi]$. Thus

$$
\lim _{\varepsilon \rightarrow 0} \int_{0}^{2 \pi} \log \left(\frac{\sqrt{f(\theta)}}{(\sqrt{f(\theta)})+\varepsilon}\right) d \theta=0
$$

which shows $(1)^{\prime}$. By the monotone convergence theorem

$$
\lim _{\varepsilon \rightarrow 0} \int_{0}^{2 \pi} \frac{d G(\theta)}{((\sqrt{f(\theta)})+\varepsilon)^{2}}=\int_{0}^{2 \pi} \frac{d G(\theta)}{f(\theta)},
$$

which shows (2)'. Finally by the bounded convergence theorem

$$
\lim _{\varepsilon \rightarrow 0} \int_{0}^{2 \pi}\left|\frac{\sqrt{f(\theta)}}{\sqrt{f(\theta)}+\varepsilon}-1\right|^{2} d G(\theta)=0,
$$

which shows (3)' and completes the proof.

The preceding lemma was needed to complete the proof of the lower bound in Theorem 2.8. We now prove a lemma providing what was needed for the upper bound.

Lemma 3.10. Let $H_{f_{N}}(R)$ be given by (3.20) and $H_{f}(R)$ be given by (1.9). Then for any $l>0$,

$$
\bigcup_{N=1}^{\infty}\left\{R \in \underline{M}_{S}: H_{f_{N}}(R) \leqq l\right\}
$$

is tight in $\underline{M}_{S}$.

If $\left\{R_{N}\right\}$ is any sequence in $\underline{M}_{S}$ converging weakly to $R \in \underline{M}_{S}$, then

$$
\varliminf_{N \rightarrow \infty} H_{f_{N}}\left(R_{N}\right) \geqq H_{f}(R) .
$$


Proof. From (2.3) we have that $f_{N}(\theta) \rightarrow f(\theta)$ uniformly in [0, 2 $]$. From Lemma 3.8 we have that $\lim _{N \rightarrow \infty} \int_{0}^{2 \pi} \log f_{N}(\theta) d \theta=\int_{0}^{2 \pi} \log f(\theta) d \theta$. Since

$$
\begin{aligned}
H_{f_{N}}(R)= & E^{R}\left\{\int_{-\infty}^{\infty} r(y \mid \omega) \log r(y \mid \omega) d y\right\} \\
& +\frac{1}{2} \log 2 \pi+\frac{1}{4 \pi} \int_{0}^{2 \pi} \frac{d G(\theta)}{f_{N}(\theta)}+\frac{1}{4 \pi} \int_{0}^{2 \pi} \log f_{N}(\theta) d \theta
\end{aligned}
$$

and

$$
\begin{aligned}
H_{f}(R)= & E^{R}\left\{\int_{-\infty}^{\infty} r(y \mid \omega) \log r(y \mid \omega) d y\right\} \\
& +\frac{1}{2} \log 2 \pi+\frac{1}{4 \pi} \int_{0}^{2 \pi} \frac{d G(\theta)}{f(\theta)}+\frac{1}{4 \pi} \int_{0}^{2 \pi} \log f(\theta) d \theta
\end{aligned}
$$

it suffices, in order to show (3.30) and (3.31), to show

$$
\bigcup_{N=1}^{\infty}\left\{R \in \underline{M}_{S}: \Phi_{N}(R) \leqq l\right\}
$$

is tight in $\underline{M}_{S}$, and, for any sequence $\left\{R_{N}\right\}$ converging to $R$,

$$
\varliminf_{N \rightarrow \infty} \Phi_{N}\left(R_{N}\right) \geqq \Phi(R),
$$

where

$$
\Phi_{N}(R)=E^{R}\left\{\int_{-\infty}^{\infty} r(y \mid \omega) \log r(y \mid \omega) d y\right\}+\frac{1}{4 \pi} \int_{0}^{2 \pi} \frac{d G(\theta)}{f_{N}(\theta)}
$$

and

$$
\Phi(R)=E^{R}\left\{\int_{-\infty}^{\infty} r(y \mid \omega) \log r(y \mid \omega) d y\right\}+\frac{1}{4 \pi} \int_{0}^{2 \pi} \frac{d G(\theta)}{f(\theta)} .
$$

We start with Lemma 3.6 where the $\phi$ of that lemma is $r(y \mid \omega)$, giving us for any $\alpha>0$,

$$
E^{R}\left\{\int_{-\infty}^{\infty} r(y \mid \omega) \log r(y \mid \omega) d y\right\} \geqq-\frac{1}{2} \log 2 \pi \alpha-\frac{1}{2 \alpha} \cdot \frac{1}{2 \pi} \int_{0}^{2 \pi} d G(\theta) .
$$

Since $f_{N}(\theta) \rightarrow f(\theta)$, there exists a $c$ such that $f_{N}(\theta) \leqq c$ on $[0,2 \pi]$. Thus, from (3.34)

$$
\begin{aligned}
E^{R}\left\{\int_{-\infty}^{\infty} r(y \mid \omega) \log r(y \mid \omega) d y\right\} & \geqq-\frac{1}{2} \log 2 \pi \alpha-\frac{1}{2 \alpha} \cdot \frac{1}{2 \pi} \int_{0}^{2 \pi} \frac{f_{N}(\theta) d G(\theta)}{f_{N}(\theta)} \\
& \geqq-\frac{1}{2} \log 2 \pi \alpha-\frac{1}{2 \alpha} \frac{c}{2 \pi} \int_{0}^{2 \pi} \frac{d G(\theta)}{f_{N}(\theta)}
\end{aligned}
$$

In this last if we let $\alpha=c / \varepsilon$ for $0<\varepsilon<1$, we get

$$
E^{R}\left\{\int_{-\infty}^{\infty} r(y \mid \omega) \log r(y \mid \omega) d y\right\} \geqq-\frac{1}{2} \log \frac{2 \pi c}{\varepsilon}-\frac{\varepsilon}{4 \pi} \int_{0}^{2 \pi} \frac{d G(\theta)}{f_{N}(\theta)},
$$


so that if $\Phi_{N}(R) \leqq l$, then

$$
\frac{1}{4 \pi} \int_{0}^{2 \pi} \frac{d G(\theta)}{f_{N}(\theta)} \leqq \frac{l+\frac{1}{2} \log \frac{2 \pi c}{\varepsilon}}{1-\varepsilon}
$$

Since $f_{N}(\theta)$ is bounded by $c$ on $[0,2 \pi]$, this last implies

$$
\frac{1}{2 \pi} \int_{0}^{2 \pi} d G(\theta) \leqq c\left(\frac{l+\frac{1}{2} \log \frac{2 \pi c}{\varepsilon}}{1-\varepsilon}\right) .
$$

This means

$$
E^{R}\left\{\omega^{2}(0)\right\} \leqq c\left(\frac{l+\frac{1}{2} \log \frac{2 \pi c}{\varepsilon}}{1-\varepsilon}\right),
$$

and since the constant on the right depends only on $l$ and not on $N$ or $R$, we see that $\bigcup_{N=1}^{\infty}\left\{R \in \underline{M}_{S}: \Phi_{N}(R) \leqq l\right\}$ is tight, which is (3.22).

To prove (3.23) we want to show that if $\left\{R_{N}\right\}$ converges weakly to $R$ and $\Phi_{N}\left(R_{N}\right)$ $\leqq M$, then $\Phi(\mathrm{R}) \leqq \mathrm{M}$.

We just proved that if $\Phi_{N}(R) \leqq l$ then $E^{R}\left\{\omega^{2}(0)\right\}$ is bounded by a constant independent of $N$. Thus if $\Phi_{N}\left(R_{N}\right) \leqq M$, we conclude that the second moments of $\left\{R_{N}\right\}$ are uniformly bounded which in turn implies the total mass of the spectral measure of $R_{N}$ is uniformly bounded, i.e., $\int_{0}^{2 \pi} d G_{N}(\theta)$ is uniformly bounded. Hence, by Helly's theorem (choosing a subsequence if necessary) $\left\{G_{N}\right\}$ converges weakly to $G^{\prime}=G+G_{1}$, where $G$ is the spectral measure of $R$ and $G_{1}$ is nonnegative.

By hypothesis

$$
\Phi_{N}\left(R_{N}\right)=E^{R_{N}}\left\{\int_{-\infty}^{\infty} r_{N}(y \mid \omega) \log r_{N}(y \mid \omega) d y\right\}+\frac{1}{4 \pi} \int_{0}^{2 \pi} \frac{d G_{N}(\theta)}{f_{N}(\theta)} \leqq M .
$$

In (3.1) if, instead of entropy of $R$ with respect to the stationary process generated by independent Gaussian, mean 0 variance 1 variables, we let $I_{\lambda}(R)$ be entropy of $R$ with respect to the same stationary Gaussian process but with variance $\lambda$ for $\lambda>0$, then, of course,

$$
I_{\lambda}(R)=E^{R}\left\{\int_{-\infty}^{\infty} r(y \mid \omega) \log r(y \mid \omega) d y\right\}+\frac{1}{2} \log 2 \pi \lambda+\frac{1}{2 \lambda} E^{R}\left\{\omega^{2}(0)\right\} .
$$

From (3.35), we see that for any $\lambda>0$,

$$
I_{\lambda}\left(R_{N}\right)+\frac{1}{4 \pi} \int_{0}^{2 \pi} \frac{d G_{N}(\theta)}{f_{N}(\theta)} \leqq M+\frac{1}{2} \log 2 \pi \lambda+\frac{1}{2 \lambda} E^{R_{N}}\left\{\omega^{2}(0)\right\} .
$$

Now, $I_{\lambda}(R)$ is lower semicontinuous on $\underline{M}_{S}$, and so since $R_{N} \Rightarrow R, f_{N}(\theta) \rightarrow f(\theta)$, $G_{N} \Rightarrow G^{\prime}=G+G_{1}$, we conclude that

$$
I_{\lambda}(R)+\frac{1}{4 \pi} \int_{0}^{2 \pi} \frac{d G^{\prime}(\theta)}{f(\theta)} \leqq M+\frac{1}{2} \log 2 \pi \lambda+\frac{1}{4 \pi \lambda} \int_{0}^{2 \pi} d G^{\prime}(\theta) .
$$


Therefore, using the expression for $I_{\lambda}(R)$,

$$
\begin{aligned}
E^{R}\left\{\int_{-\infty}^{\infty} r(y \mid \omega) \log r(y \mid \omega)\right\} \\
\quad+\frac{1}{2} \log 2 \pi \lambda+\frac{1}{4 \pi \lambda} \int_{0}^{2 \pi} d G(\theta)+\frac{1}{4 \pi} \int_{0}^{2 \pi} \frac{d G^{\prime}(\theta)}{f(\theta)} \\
\leqq M+\frac{1}{2} \log 2 \pi \lambda+\frac{1}{4 \pi \lambda} \int_{0}^{2 \pi} d G^{\prime}(\theta) .
\end{aligned}
$$

Since this last is true for any $\lambda>0$, we let $\lambda \rightarrow \infty$, after cancelling $\frac{1}{2} \log 2 \pi \lambda$ from both sides, to obtain

$$
E^{R}\left\{\int_{-\infty}^{\infty} r(y \mid \omega) \log r(y \mid \omega) d y\right\}+\frac{1}{4 \pi} \int_{0}^{2 \pi} \frac{d G^{\prime}(\theta)}{f(\theta)} \leqq M .
$$

Since $G^{\prime}(\theta)=G(\theta)+G_{1}(\theta)$ and $G_{1}(\theta)$ is nonnegative, we conclude that in fact

$$
\Phi(R)=E^{R}\left\{\int_{-\infty}^{\infty} r(y \mid \omega) \log r(y \mid \omega) d y\right\}+\frac{1}{4 \pi} \int_{0}^{2 \pi} \frac{d G(\theta)}{f(\theta)} \leqq M,
$$

which completes the proof.

\section{References}

1. Donsker, M.D., Varadhan, S.R.S.: Asymptotic evaluation of certain Wiener integrals for large time. Functional Integration and its Applications, Proceedings of the International Conference held at the Cumberland Lodge, Windsor Great Park, London, in April 1974, A. M. Arthurs, (ed.). Oxford: Clarendon 1975

2. Donsker, M.D., Varadhan, S.R.S.: Asymptotic evaluation of certain Markov process expectations for large time, I. Commun. Pure Appl. Math. 28, 1-47 (1976); II. Commun. Pure Appl. Math. 28, 279-301 (1975); III. Commun. Pure Appl. Math. 29, 389-461 (1976); IV. Commun. Pure Appl. Math. 36, 183-212 (1983)

3. Donsker, M.D., Varadhan, S.R.S.: Asymptotics for the Wiener Sausage. Commun. Pure Appl. Math. 28, 525-565 (1975)

4. Donsker, M.D., Varadhan, S.R.S.: On laws of the iterated logarithm for local times. Commun. Pure Appl. Math. 30, 707-753 (1977)

5. Donsker, M.D., Varadhan, S.R.S.: Asymptotics for the Polaron. Commun. Pure Appl. Math. 36, 505-528 (1983)

6. Orey, S.: Large deviations and Shanon-McMillan theorems (preprint)

Communicated by A. Jaffe

Received July 6, 1984 